\title{
Interactive comment on "4D dispersion of total gaseous mercury derived from a mining source: identification of criteria to assess risks related with high concentrations of atmospheric mercury" by José M. Esbrí et al.
}

José M. Esbrí et al.

josemaria.esbri@uclm.es

Received and published: 19 May 2020

The manuscript studies the alternatives that exist to make monitoring works in an area contaminated with anthropogenic gaseous mercury. It recommends measurements at different heights, over significant transects and the repetition of these measurements over time. Although the results seem to be appropriate for a complex area such as the one they have chosen as study area, the effort involved in obtaining this minimum number of data is great and perhaps could be simplified if a previous study were made of the most important factors involved in the local cycle of mercury, in a short

Printer-friendly version

Discussion paper 
period. In any case, the manuscript presents a monitoring option that seems to offer very significant data and that could be applicable to any contaminated area. Lines 44-47. Definition of TGM include wrongly particle-bound mercury fraction. Revise it. Done. The new version explains this with the following sentence: GEM and RGM together these species constitute 'total gaseous mercury' (TGM). Lines 51-52. If water is included in this transfer pathways, what about sediments? Thanks for the comment. In principle, we did not consider it important to include sediments in this introduction, since we were going to study mercury emissions in an urban environment, but it is true that the results have shown that sediments are an important source of emission to consider in the area of study. For these reasons, we have included it in the following sentence: Numerous $\mathrm{Hg}$ transfer pathways are involved in this cycle, and these include soil-atmosphere, soil-plant, plant-atmosphere, and water-atmosphere, and sedimentswater, amongst others. Line 61. Again sediments are missing. . . It is difficult to find references including data about gaseous mercury emissions from sediments, so we have included sediments with a reference to emissions from salt marshes, which are not a perfect analogue, but which well expresses the idea of the timing of mercury emissions in a river context. The new sentence is as follows: A maximum emission during diurnal hours was described for soils (Zhu et al., 2015), mine materials (Eckley et al., 2011), waters (O'Driscoll et al., 2003), sediments (Sizmur et al., 2017) and snow (Maxwell et al., 2013), while forb leaf (Stamenkovic et al., 2008) and growing broad leaf (Fu et al., 2016) reach their minimum emission rates during diurnal hours. Line 100. Explain what are the sources of medium importance, polluted wastes? Ore outcrops? Done. The new sentence is as follows: In the town centre of Almadenejos there are four emission sources of medium importance (cinnabar wastes), while in the vicinity there is one of very high importance (MMP), one of high importance (Nueva Concepción mine), and two of low importance (a contaminated road running North of the town and the course of the Valdeazogues river, since it passes through the El Entredicho mine). Lines 128-131. Then there is no soil data, why? The monitoring strategy was designed to study the vertical flows of mercury from dispersed emission

Interactive comment

Printer-friendly version

Discussion paper

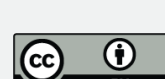


sources in the urban area, not to study emissions from the soils of the Almadenejos treatment plant, which are not contaminated. For this reason, the possibility of monitoring the temperature and moisture of these soils was not considered necessary. Line 147. Add a comma in 3,650 and unifies the way the figures are represented throughout the manuscript Done. Lines 181-183. Add a reference to support this sentence. We have not fully understood this suggestion. The sentence has a first reference to the wastes that exist in the metallurgical complex, which could be referenced by the already mentioned Martinez-Coronado et al. (2011), and a second part that describes minor sources identified in this work, and that is why we do not refer to them. We have added the reference to the first part of the sentence. Line 210 . There is no reference in the methodology section to soil temperature measurements, explain this. These soil temperature data are used as reference but do not belong to this work, but to a previous one in which a nearby soil was monitored to study its emissions. In the present work, these data are used as general trends that are expected to continue year after year without significant changes. Unfortunately, the work remains unpublished and we have not been able to reference it. Line 250. Something is missed in the top of the figure, in the scale bars Done. The figure has been edited by a previous suggestion of reviewer 1 to solve this problem. Line 287. In Figure 7, is it possible to separate transitional populations in the spring charts of profiles 1 and 3 ? Thanks for the suggestion. Although the trend seems to change, there is not a sufficient number of data to establish an anomaly threshold. Lines 307-312. The topographic profile is not enough to understand this, how are the river valleys? open or narrow? what is the difference in heights from the nearby mountains? and the slopes? Done. A new sentence explaining this has been added: It is necessary to emphasize that the topography of the study area consists of mountainous alignments of smooth slopes, typical of the Appalachian relief, with maximum differences of heights of 220 meters. Line 375 . Indicate in the figure the inhabited area where risk from chronic exposure may occur Done. The manuscript has a revised version of the figure highlighting the inhabited area Line 515 . Unify the decimals in the numbers Done. 
Interactive comment on Atmos. Chem. Phys. Discuss., https://doi.org/10.5194/acp-2019-1107, 2020.
ACPD

Interactive comment 


\section{ACPD}

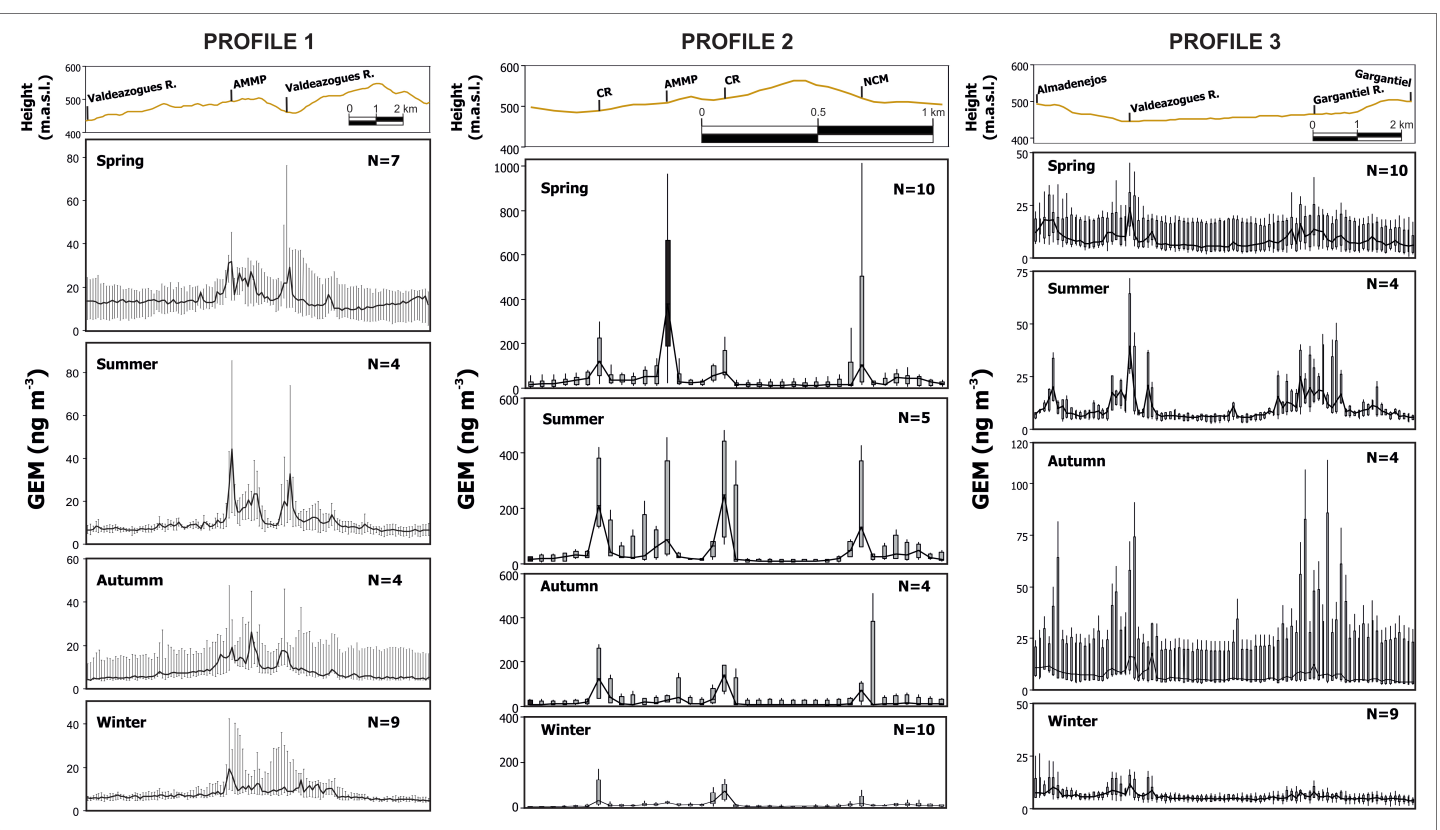

Interactive

comment

Fig. 1. 


\title{
ACPD
}

\author{
Interactive \\ comment
}

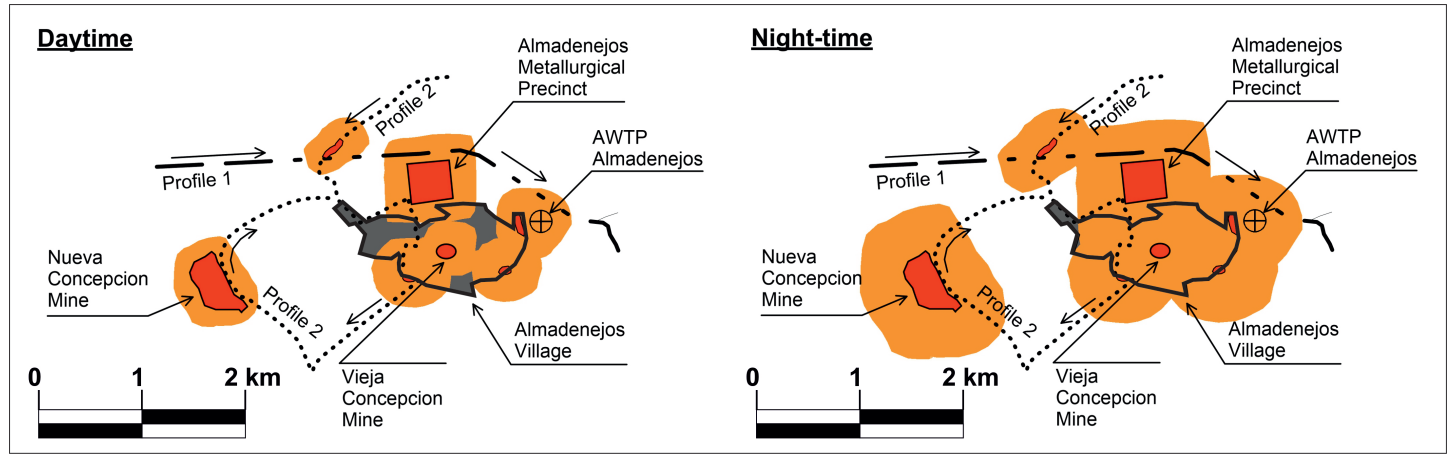

Fig. 2.

Printer-friendly version

Discussion paper 